\title{
Propuesta metodológica para la generación de zonas de asignación escolar: fomento de una movilidad sostenible y activa
}

\author{
Francisco Javier Jaraíz-Cabanillas \\ Ana María Hernández-Carretero
}

Universidad de Extremadura. Departamento de Didáctica de las Ciencias Sociales

jfjaraiz@unex.es

ahernand@unex.es

Enrique Eugenio Ruiz-Labrador

José Antonio Gutiérrez-Gallego

Universidad de Extremadura. Departamento de Expresión Gráfica

eruizl@unex.es

jagutier@unex.es

\section{Resumen}

El objetivo principal de este trabajo es identificar los modos de transporte utilizados en el acceso a los centros educativos por parte de la comunidad escolar (alumnos y profesores) y desarrollar una metodología con la que generar áreas de asignación escolar a cada centro en función del número de plazas ofertadas y la distancia de la residencia de los alumnos al mismo caminando. De este modo, se busca fomentar unos hábitos de movilidad más sostenibles y seguros. Las fuentes empleadas en este estudio son las encuestas realizadas a los alumnos, la información extraída del padrón municipal de habitantes y la proporcionada por el Centro de Profesores y Recursos. Las encuestas permiten conocer los hábitos de movilidad, mientras que el resto de información se emplea para la generación de las áreas de asignación haciendo uso de herramientas SIG. El ámbito de estudio es la ciudad de Mérida, Extremadura.

Palabras clave: movilidad escolar; encuestas; zonas de asignación; sistemas de información geográfica (SIG) 
Resum. Proposta metodològica per a la generació de zones d'assignació escolar: foment d'una mobilitat sostenible $i$ activa

L'objectiu principal d'aquest treball és identificar els modes de transport utilitzats en l'accés als centres educatius per part de la comunitat escolar (alumnes i professors) i desenvolupar una metodologia per generar àrees d'assignació escolar a cada centre en funció del nombre de places ofertes i de la distància caminant des de la residència dels estudiants a l'escola. Per tant, pretén promoure els hàbits de mobilitat més sostenibles i segurs. Les fonts utilitzades en aquest estudi són les enquestes als estudiants, informació extreta del padró municipal d'habitants i la proporcionada pel Centre de Professors i Recursos. Les enquestes ens permeten conèixer els hàbits de mobilitat, mentre que la resta de la informació s'empra per a la generació de les àrees d'assignació amb una eina SIG. L'anàlisi s'ha aplicat al cas de la ciutat de Mèrida, Extremadura.

Paraules clau: mobilitat escolar; enquestes; zones d'assignació; sistemes d'informació geogràfica (SIG)

Résumé. Une proposition méthodologique pour la création des secteurs de recrutement scolaire: promotion d'une mobilité active et durable

L'objectif principal de ce travail est d'identifier les modes de transport utilisés dans l'accès aux écoles de la communauté scolaire (élèves et enseignants) et de développer une méthodologie pour générer des zones d'attribution à chaque centre scolaire, en fonction du nombre de places à l'école et de la distance de marche avec le lieu de résidence des élèves. Ainsi, on cherche promouvoir des habitudes plus sûres et une meilleure mobilité. Les sources utilisées dans cette étude sont une enquête réalisée auprès d'élèves, des informations extraites du registre municipal des habitants et fournies par le centre local de ressources et d'enseignants. Cette enquête permet de connaître les habitudes de mobilité, alors que d'autres informations ont été utilisées pour la génération zones d'attribution, avec l'aide des outils SIG. L'étude a été réalisée dans la ville de Merida (sud-ouest de l'Espagne).

Mots-clés: mobilité de l'école; enquêtes; cartographie; systèmes d'information géographique (SIG)

Abstract. A methodological proposal to plan school catchment areas: Promoting active and sustainable mobility

The main aim of this paper is to offer a local-level planning proposal in order to improve daily mobility to school by promoting road safety and more sustainable habits among children. The case of the city of Mérida (southwest of Spain) has been analysed. The sources employed were a survey of students, as well as data from the municipal register, the public transport company and the local Teachers and Resources Centre. The survey was critical in determining mobility habits and students' perceptions, while the rest of the information was used to design a school mobility plan. Using GIS tools, a new set of school catchment areas were identified in order to increase road safety and reduce travel costs.

Keywords: School mobility; surveys; assignment areas; Geographical Information Systems (GIS) 


\section{Sumario}
1. Introducción
4. Análisis y resultados
2. Área de estudio
5. Discusión y conclusiones
3. Metodología
Referencias bibliográficas

\section{Introducción}

En los últimos cincuenta años las ciudades europeas han copiado el modelo de crecimiento urbano anglosajón, caracterizado por una expansión cada vez mayor hacia zonas periféricas y alejándose del centro urbano primigenio. Esto provoca una descentralización de servicios (entre los que se encuentran los educativos) y una especialización de usos (industriales, recreativos, residenciales, etc.), ubicados en entornos cercanos a las principales vías de comunicación (Brueckner, 2000; Dombriz, 2009). Esta tendencia ha ido paralela además a un cambio en las pautas de movilidad de sus usuarios (Seguí y Martínez, 2004; Monzón, 2005; Pozueta y Gurovich, 2007): se detecta un aumento exponencial del número y la longitud de los desplazamientos (Steg y Gifford, 2005; Ortúzar y Willumsen, 2008) junto a un uso abusivo del vehículo privado con ocupaciones bajas (Dombriz, 2009). Así, este modelo de movilidad está muy encauzado al uso de modos de transporte motorizados para llevar a cabo buena parte de los movimientos diarios, entre los que destacan los denominados obligados, es decir, aquellos que se realizan por motivos de estudio o trabajo (Ortúzar y Willumsen, 2008).

Estas nuevas pautas de movilidad provocan ciertos problemas: congestión vehicular (Cameron et al., 2003), contaminación acústica y atmosférica (Barr y Prillwitz, 2012), disminución de la seguridad en los desplazamientos (Hadayeghi et al., 2003), complicaciones a la hora de estacionar e incremento de problemas de salud y de índole social (Bocarejo y Oviedo, 2012).

Para mitigar tales problemas y conseguir una movilidad más sostenible, la Unión Europea lleva instando, desde finales del siglo XX, a todos los agentes públicos a que implementen actuaciones que faciliten este cambio de movilidad en beneficio de un mayor bienestar sobre la población residente (CEC, 2006, 2007, 2011): un reparto modal que dé más peso a los modos de transporte colectivos y sostenibles, reducción de emisiones de gases de efecto invernadero, disminución de la contaminación acústica y aumento de la equidad social en el acceso a todos los bienes y servicios. Resulta clave, por lo tanto, fomentar una buena educación vial para los alumnos, tanto desde la educación reglada como desde la no reglada. Su importancia es tal que, de ser tratada como un tema transversal en la educación, ha pasado a recogerse como un objetivo primordial para la educación primaria y secundaria, centrándose no solo en el conocimiento y cumplimiento de las normas básicas como peatones y usuarios, sino también en el ahorro energético. 
Dada la importancia que ha cobrado el problema de la movilidad segura en el acceso al entorno educativo y el hecho de que el $58 \%$ de los desplazamientos detectados en población con edades comprendidas entre los 6 y los 20 años son por motivos de estudios (Ministerio de Fomento, 2007), resulta clave diagnosticar las pautas de desplazamiento seguidas por los alumnos en dicho acceso y considerar al mismo tiempo su coincidencia en el tiempo y el espacio con otros flujos urbanos (Cardozo et al., 2012). De esta forma, se busca convertir el itinerario escolar habitual en un entorno adaptado definido por un acceso seguro y autónomo al centro educativo, que mejore la salud (McDonald, 2007a; Andersson et al., 2012) y la socialización de los alumnos (Davis y Jones, 1997; Collins y Kearns, 2001), junto a la disponibilidad de espacios verdes y servicios básicos que favorezcan el desarrollo de actividades diversas minimizando la contaminación ambiental (Collins y Kearns, 2001; Kyttä, 2008; Broberg et al., 2013).

El incremento exponencial del tráfico rodado en los entornos educativos y la puesta en marcha de políticas liberales para la localización de centros educativos en las áreas de extrarradio (Davis y Jones, 1997; Collins y Kearns, 2001) y para la elección del centro en el que cursar los estudios provocan cierto grado de inseguridad entre los padres, cuya consecuencia es la privación a sus hijos del derecho a la movilidad autónoma. Esta política liberal sustituye a una anterior más tradicional, caracterizada por una asignación de estudiantes a centros educativos en función de la localización de sus lugares de residencia (Andersson et al., 2012). Aunque este tipo de asignación es más racional desde el punto de vista de la movilidad, suele incumplirse con frecuencia, ya que se observa desconfianza de nuevo por parte de los padres hacia los colectivos acogidos en los centros, la ubicación de los mismos o la diferencia de estatus social entre públicos y concertados.

Además, los modelos actuales de asignación de alumnos a los centros no ayudan a reducir tales problemas, sino todo lo contrario. De ahí que sea necesaria una nueva propuesta de asignación, junto a la implantación de itinerarios escolares seguros, como vía para aumentar el derecho a la libre movilidad en los chicos y una vuelta a la racionalidad en los desplazamientos (Martin y Carlson, 2005; Kerr et al., 2006; McMillan, 2007; Napier et al., 2011).

Una buena opción para fomentar este nuevo modelo de movilidad autónoma en desplazamientos de cierta distancia (es decir, superior a los veinte minutos) es el transporte público, el cual proporciona seguridad y confortabilidad en los chicos que pretenden viajar hacia y desde el colegio a sus lugares de residencia, pero que es poco utilizado (Roberts et al., 1996, Davis y Jones, 1997). Esta infrautilización del transporte público puede reflejar una planificación y unas rutas poco convenientes, un aumento de la preocupación por la seguridad de los niños y el aumento de las tasas de uso y tenencia del vehículo privado (Roberts et al., 1997; Collins y Kearns, 2001).

Con respecto a las propuestas de asignación, debe dársele más peso a la distancia de desplazamiento entre áreas residenciales y centros educativos. Sin embargo, un análisis sencillo de esta no ofrece información precisa sobre la 
elección real, ya que está influida por factores geográficos como la ubicación del centro en relación con el lugar de residencia o la oferta de transporte (Andersson et al., 2012). Además, existen pocos estudios dirigidos directamente a los análisis de la capacidad o posibilidad de superar las restricciones de espacio para conservar una elección ilimitada (Trumberg, 2011).

La distancia al centro, por consiguiente, se ve muy influenciada por una serie de variables: 1) el estatus social, que influye en la capacidad de los individuos de aumentar el coste de la distancia; 2) la raza o etnia de la unidad familiar; 3) el nivel educativo de los padres (vinculado al estatus socioeconómico) (Karsten et al., 2003; Andre-Bechely, 2007; Chen et al., 2011; Sang et al., 2011). En este sentido, hay estudios que muestran que estudiantes cuyas familias tienen un nivel educativo alto realizan viajes de mayor distancia para desplazarse al centro educativo (Bunar y Kallstenius, 2005), mientras que los centros con menor rendimiento académico reclutan a estudiantes que residen en un área de influencia más cercana (Walford, 2003; Butler et al., 2007; Trumberg, 2011). Y finalmente, 4) la percepción de los alumnos acerca del automóvil heredada de sus padres (Mackett, 2003; Jensen, 2008; Fyhri et al., 2011; Deka, 2013), independientemente de su nivel académico (Mackett, 2002; Jensen et al., 2004; Mackett et al., 2005).

Entre las consecuencias negativas que afectan directamente a los jóvenes, destacan el aumento de sobrepeso (Fox, 2004; Salmon et al., 2005) y la pérdida de independencia e interrelación con el entorno urbano (Fotel y Thomsen, 2004). En cambio, los beneficios derivados de una movilidad independiente y sostenible son varios: desarrollo de habilidades motoras e incremento de actividad física o mejora cognitivo-social (Brown et al., 2008). Por todo ello, resulta imprescindible identificar las pautas de movilidad adquiridas en este tipo de desplazamientos y concienciar de los beneficios de una movilidad más sostenible y segura.

Por consiguiente, considerando todo lo anterior, los objetivos específicos que se desarrollarán en el área de estudio que supone la ciudad de Mérida (Extremadura) son: 1) detectar el modo de transporte utilizado por padres y alumnos para acceder al centro educativo e identificar al mismo tiempo alguna cuestión interesante, como la percepción que tienen los alumnos de la distancia en dichos desplazamientos o sus propias preferencias en cuanto al modo idóneo para realizarlos, y 2) proponer una metodología que permita generar áreas de asignación sostenibles del alumnado demandante a los diferentes centros educativos ofertados. Es necesario aclarar que la percepción de la distancia revelada por los alumnos en el proceso de encuesta no es el dato utilizado posteriormente en la propuesta de asignación. Solo se tiene en cuenta para detectar cómo, en muchos casos, el modo realmente utilizado no es el preferido por los alumnos. En el proceso de zonificación se utiliza la localización real de los residentes de Mérida en edad escolar (Padrón Municipal de Habitantes del Ayuntamiento de Mérida, 2012). Con todo ello, se busca fomentar unos hábitos de movilidad más sostenibles y seguros, mejorar las relaciones sociales entre los jóvenes y orientarlos hacia unas prácticas más saludables de desplazamiento. 


\section{2. Área de estudio}

Se plantea una investigación que se enmarca en la ciudad de Mérida (figura 1), cuya población es de 57.173 habitantes (INE, 2012²) y su extensión urbana de poco más de $16 \mathrm{~km}^{2}$, como ejemplo para aplicar esta propuesta metodológica en ciudades de tipo medio. Su enclave estratégico (entre las dos vías vertebradoras del territorio extremeño), unido a sus roles administrativo y turístico, hace que soporte un volumen de desplazamientos muy elevado, tanto de origen interno (residentes) como externo (población que accede diariamente por motivos burocráticos, de trabajo, estudios, turísticos o de ocio, entre otros). Así, esta ciudad soporta un flujo diario que ronda los 26.600 viajes, de los cuales el $10 \%$ se realizan en las horas punta (de 8 a 9 h y de 14 a 15 h) y de estos últimos, el $32 \%$ corresponden a desplazamientos vinculados a motivos de acceso a centros educativos (Gutiérrez y Pedrazo, 2010).

En lo que tiene que ver con la oferta educativa de la ciudad, esta consta de 27 centros repartidos por todo el entorno urbano, clasificados entre educación primaria y secundaria. De estos, 20 son centros públicos y 7 , concertados, que imparten tanto educación primaria como secundaria. Esta oferta educativa pre-

Figura 1. Mapa localizador de la ciudad de Mérida

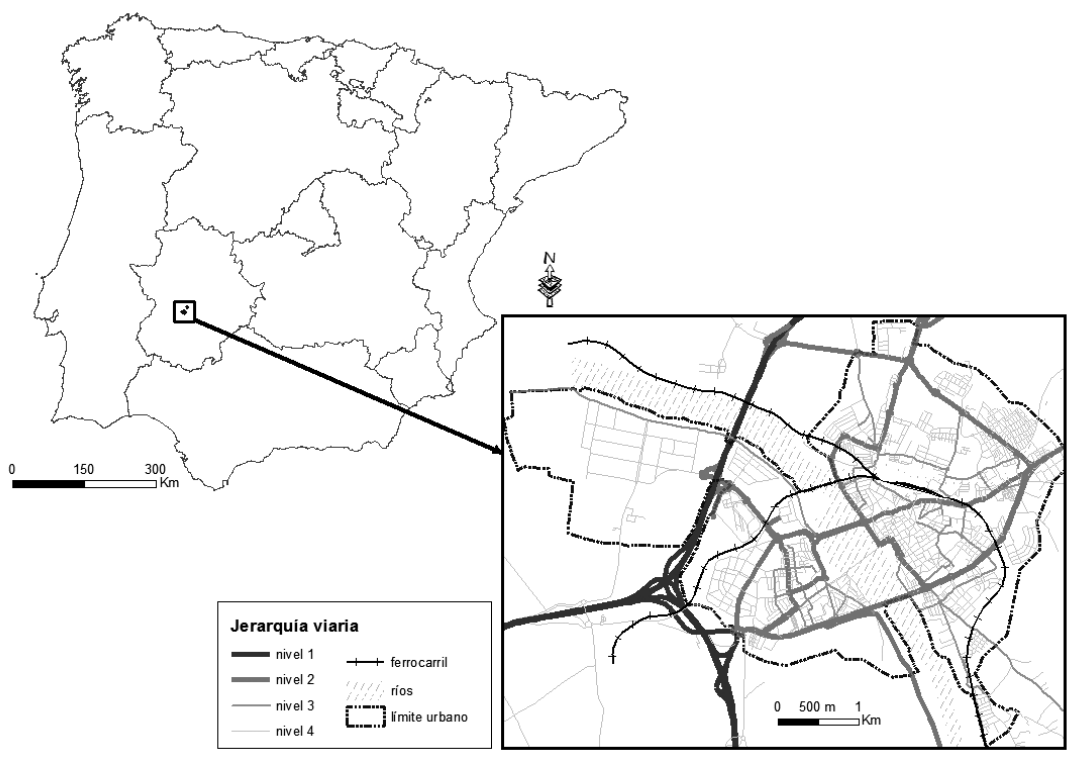

Fuente: elaboración propia.

1. Padrón Municipal de Habitantes del año 2012, Instituto Nacional de Estadística (INE). 
Figura 2. Áreas de asignación actual de estudiantes a los centros educativos emeritenses

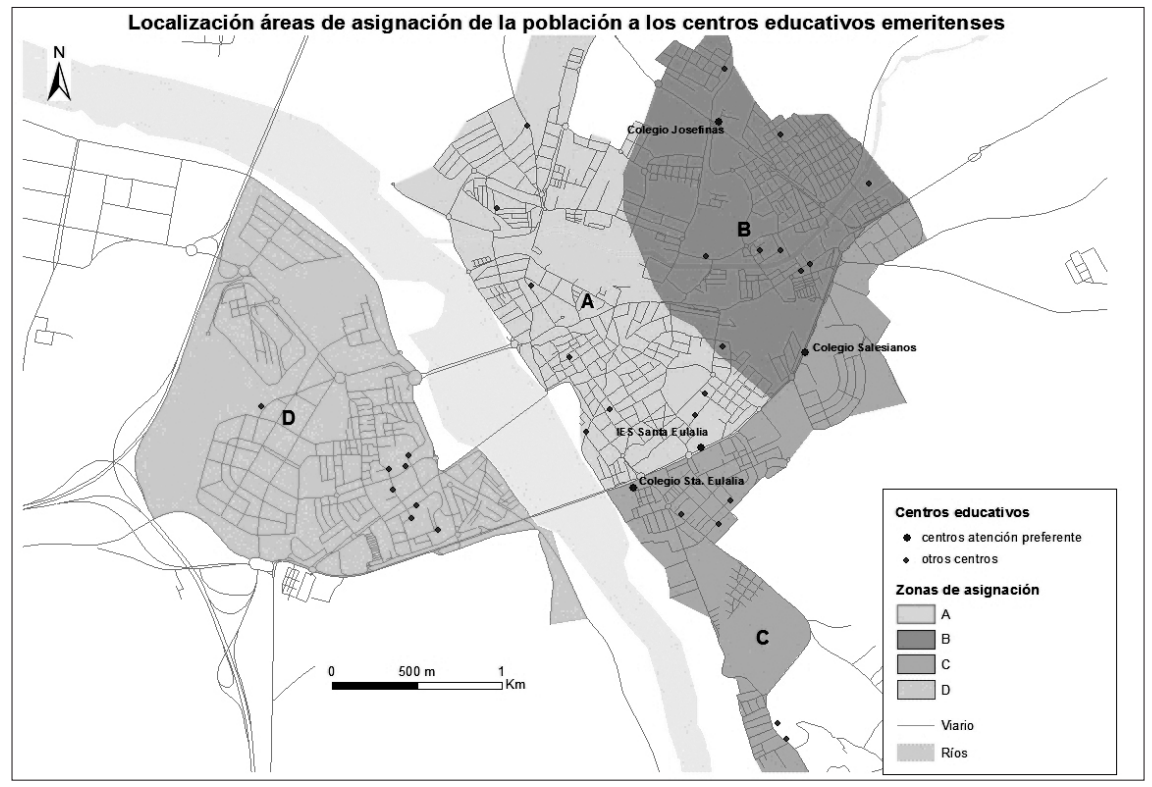

Fuente: elaboración propia.

senta unas áreas de asignación que, hasta la fecha, se distinguen por el enorme espacio que abarcan. Esto concede una libertad de elección que incita a utilizar modos de transporte poco sostenibles en el acceso a estos centros (figura 2).

La ubicación de los cuatro centros con un mayor volumen de alumnos (21\% de los alumnos emeritenses) en las vías con mayor intensidad vehicular en hora punta (más de 1.200 vehículos/hora), a los que habría que sumar seis centros más ubicados en las proximidades de los primeros (41\% del alumnado) y la coexistencia de los desplazamientos escolares con buena parte de las actividades económicas, provoca problemas de congestión vehicular y falta de seguridad en las inmediaciones (figura 3). La deficiente planificación de estacionamientos y el escaso uso de los modos sostenibles agravan este problema.

\section{Metodología}

La metodología empleada en este estudio parte de dos fuentes de datos principales: encuestas dirigidas a los alumnos de educación primaria y secundaria, y la información del padrón de habitantes del Ayuntamiento de Mérida. A través de la encuesta se determinan los modos de desplazamiento habituales de los alumnos. El padrón de habitantes permite ubicar a los alumnos que potencialmente están en edad de desplazarse a los centros educativos ofertados sobre la trama urbana de la ciudad. Con esta información se puede realizar un 
Figura 3. Localización de los centros educativos en la red e intensidades de tráfico

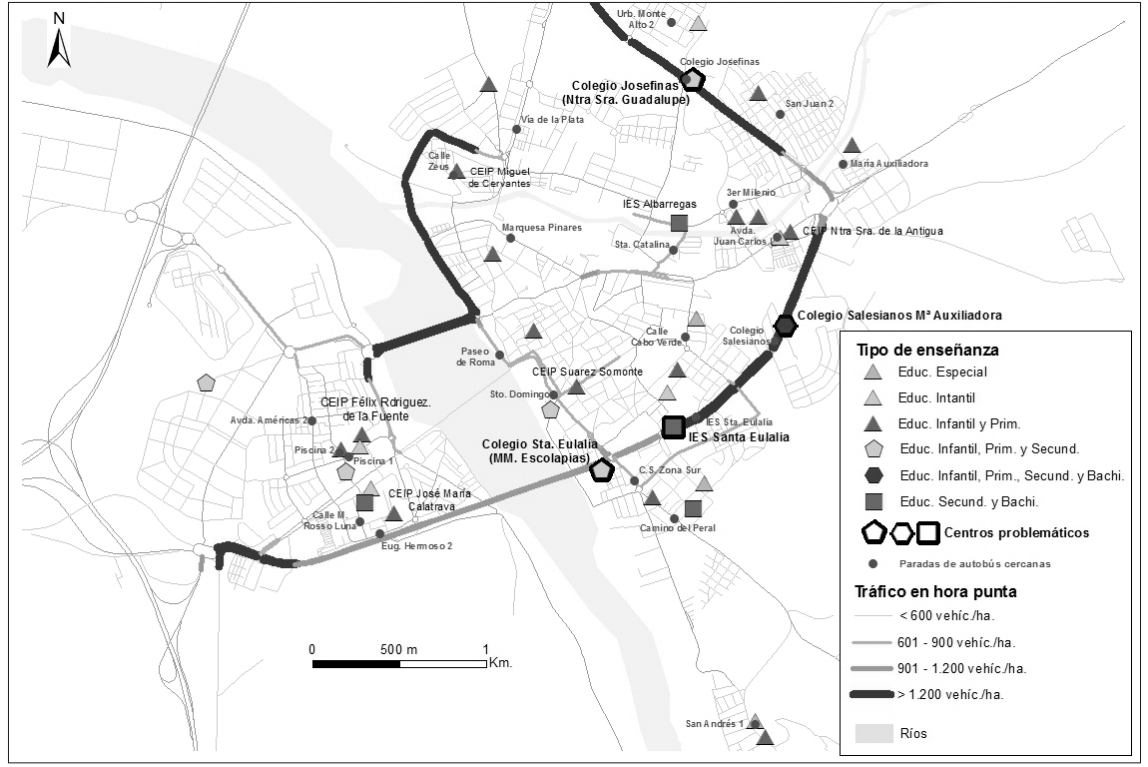

Fuente: elaboración propia.

análisis completo de la movilidad y plantear una propuesta de cambio en lo que a zonificación de asignación se refiere haciendo uso de entornos propios de los sistemas de información geográfica genéricos (en adelante SIG). A estas dos fuentes principales de datos se les une información facilitada por el Centro de Profesores y Recursos de Mérida, relativa al número de plazas ofertadas en cada centro educativo. Esta información sirve de límite en la propuesta de asignación planteada. Se describe a continuación pormenorizadamente cada grupo de tareas asociadas al proceso metodológico seguido en esta investigación.

\subsection{Encuesta de movilidad de los alumnos}

El primer paso para abordar los hábitos de movilidad existentes en las inmediaciones de los centros educativos es identificarlos y analizarlos. Para ello, se hace uso de la encuesta realizada a los alumnos. Lo más destacado de dicha encuesta es la identificación de los modos de transporte utilizados diariamente en este tipo de desplazamientos.

El método utilizado para recabar esta información es el de la autoencuesta de preferencias reveladas: se reparte en clase una plantilla de encuesta en papel a cada alumno objeto de estudio y este la rellena de forma individual. Las plantillas se realizan atendiendo al nivel educativo de los alumnos objeto de estudio. Para hacer más ameno este proceso se busca que dicha plantilla tenga 
un estilo original y de fácil comprensión por parte de los alumnos. Entre las cuestiones más importantes destacaban: ¿Cómo llegas al centro educativo?; ¿Prefieres ir en automóvil antes que caminando?; ¿Conoces el sistema de autobús urbano existente en tu ciudad?; o ¿Cuánto tiempo tardas en llegar desde tu casa al centro educativo?

Una vez recabada toda la información se genera una base de datos para extraer los resultados finales más interesantes del trabajo. En el proceso de transformación al formato digital se mantiene la misma estructura de preguntas que la encuesta en papel, lo que evita pérdidas de información.

Para el cálculo del tamaño mínimo muestral se consideró el número de alumnos matriculado en todos los centros de la ciudad como población total, teniendo en cuenta un intervalo de confianza del $95 \%$ y un error aceptable del $3 \%$. Sin embargo, la muestra final obtenida es superior a la que en un principio se obtuvo del cálculo a tenor de la población total, ya que se recogió un total de 3.396 encuestas de alumnos de entre $3^{\circ}$ de primaria y $4^{\circ}$ de ESO, repartidos en 21 centros educativos tanto públicos como concertados (tabla 1).

Tabla 1. Alumnos encuestados por centro educativo y curso

$\%$ alumnos

Centros

$3^{\circ} \mathrm{EP} 4^{\circ} \mathrm{EP} 5^{\circ} \mathrm{EP} 6^{\circ} \mathrm{EP} 1^{\circ} \mathrm{ESO} 2^{\circ} \mathrm{ESO} 3^{\circ} \mathrm{ESO} 4^{\circ} \mathrm{ESO}$ Encuestas encuestados Alumnos

\begin{tabular}{lrrrrrrrrrrr}
\hline CEIP A. Machado & 5 & 9 & 8 & 6 & - & - & - & - & 28 & $30,1 \%$ & 93 \\
CEIP Octavio Augusto & 26 & 50 & 23 & 45 & - & - & - & - & 144 & $32,14 \%$ & 448 \\
CEIP J. M. de Calatrava & 14 & 14 & 16 & 10 & - & - & - & - & 54 & $45,76 \%$ & 118 \\
CEIP Santa Eulalia & - & - & - & 58 & - & - & - & - & 58 & $8,1 \%$ & 716 \\
CEIP Giner de los Ríos & 48 & 48 & 46 & 54 & - & - & - & - & 196 & $41,61 \%$ & 471 \\
Colegio San Juan Bosco & 21 & 27 & 23 & 27 & - & - & - & - & 98 & $41,88 \%$ & 234 \\
CEIP N. S. de la Antigua & 19 & 23 & 33 & 19 & - & - & - & - & 94 & $37,6 \%$ & 250 \\
CEIP F. G. Lorca & 37 & 24 & 27 & 23 & - & - & - & - & 111 & $48,25 \%$ & 230 \\
CEIP F. R. de la Fuente & 25 & 25 & 19 & 20 & - & - & - & - & 89 & $50,27 \%$ & 177 \\
Colegio San Luis & 15 & 16 & 20 & 10 & - & - & - & - & 61 & $32,8 \%$ & 186 \\
CEIP Pablo Neruda & 7 & 8 & 5 & 6 & - & - & - & - & 26 & $36,61 \%$ & 71 \\
CEIP Juan XXIII & 13 & 1 & 12 & 10 & - & - & - & - & 46 & $47,92 \%$ & 96 \\
CEIP Trajano & & & 55 & 49 & - & - & - & - & 104 & $18,90 \%$ & 550 \\
CEIP M. de Cervantes & 23 & 24 & 17 & 23 & - & - & - & - & 87 & $29 \%$ & 300 \\
Col. María Auxiliadora & - & - & - & - & - & 81 & 74 & 69 & 224 & $24,22 \%$ & 925 \\
Coop. Santa Eulalia & 24 & 26 & 27 & 29 & 27 & 26 & 22 & 22 & 203 & $54,86 \%$ & 370 \\
IES Extremadura & - & - & - & - & 94 & 66 & 41 & 43 & 244 & $47,10 \%$ & 518 \\
IES Santa Eulalia & - & - & - & - & 148 & 90 & 107 & 118 & 463 & $34,29 \%$ & 1.350 \\
IES Emérita Augusta & - & - & - & - & - & - & 78 & 13 & 91 & $13,91 \%$ & 654 \\
IES Albarregas & - & - & - & - & 90 & 83 & - & - & 173 & $22,24 \%$ & 778 \\
Coop. Docente Atenea & 51 & 53 & 55 & 45 & 46 & 46 & 44 & 38 & 378 & $55,92 \%$ & 676 \\
TOTAL & 328 & 348 & 386 & 434 & 405 & 392 & 366 & 302 & 3.396 & $35,88 \%$ & 9.211 \\
\hline
\end{tabular}

Fuente: elaboración propia. 


\subsection{Propuesta metodológica de asignación}

Una vez identificados los modos de transporte utilizados, el porcentaje de estudiantes que acceden a centros educativos situados en vías urbanas con una elevada intensidad circulatoria (40,6 \% según la encuesta realizada y partiendo de los datos facilitados por el Centro de Recursos y Profesores de Mérida) y la zonificación de asignación actual (figura 2), el siguiente paso consiste en proponer una nueva zonificación de asignación educativa que reduzca al máximo la distancia de desplazamiento a todos los centros educativos y fomente el acceso peatonal y la movilidad autónoma de los alumnos. Así, la nueva zonificación planificada distribuye a los alumnos entre los distintos centros educativos ofertados de la ciudad en función del nivel educativo (infantil, primaria y secundaria), del volumen de plazas ofertadas en cada centro para cada uno de estos niveles y, sobre todo, de la premisa de que los alumnos deben asistir al centro más próximo a su domicilio habitual (distancia a pie calculada en minutos).

Para el desarrollo de este modelo de asignación se utiliza el entorno SIG de ESRI, ArcGis 10.1 y su extensión de análisis de redes. Junto al SIG también se utilizan aplicaciones como el sistema gestor de base de datos MS Access y la hoja de cálculo MS Excel. Para un tratamiento óptimo y ordenado de la información, se trabaja con cada nivel educativo de forma independiente. La información de partida en esta tarea es un listado de residentes facilitado por el servicio del padrón de habitantes del Ayuntamiento de Mérida. En este se muestran datos como la localización residencial, la edad y el género de forma individual. Esta información, unida al número de plazas ofertadas en cada centro educativo (Centro de Profesores y Recursos), resulta clave para generar la nueva zonificación.

Los pasos seguidos en esta propuesta metodológica parten del cálculo de una matriz de costes (matriz origen/destino), en donde se contabilizan todos los tiempos de desplazamiento peatonal a través de la red urbana existente, entre cada punto poblacional (origen del desplazamiento) y todos los centros educativos ofertados en Mérida (destinos del desplazamiento). En el caso de los orígenes, únicamente se considera a la población residente en edad de desplazarse a uno de los centros educativos en cuestión.

Una vez generada la matriz $\mathrm{O} / \mathrm{D}$, se importa a una base de datos donde se procede a calcular el volumen de alumnos que ha de ir a cada centro educativo en función del nivel ofertado y la distancia mínima de desplazamiento (en este primer cálculo no se tiene en cuenta la limitación de plazas de cada centro; esta sería la asignación óptima si no existiese tal limitación). Además de la matriz de costes, se implementan en la base de datos otras tablas con información útil para el trabajo: 1) tabla de centros educativos con los niveles que imparten y el número de plazas disponibles, 2) información poblacional del padrón municipal de habitantes facilitada por el Ayuntamiento de Mérida, con la dirección postal de cada individuo y su fecha de nacimiento (de gran utilidad para calcular la edad de los alumnos), y 3) tabla de edades individuales y su agrupación al nivel educativo correspondiente. 
Para extraer la información en la base de datos, se lleva a cabo un proceso de generación de consultas y relación entre las tablas previamente mencionadas. Los resultados se exportan a una hoja de cálculo, donde se estima el tiempo límite de cada zona de asignación (tabla 2). En las dos primeras columnas de esta hoja de cálculo se recoge el número de plazas ofertadas en cada centro y el volumen de alumnos potencialmente asignados al mismo (es decir, alumnos asignados sin considerar la limitación de plazas). A continuación, y en diferentes columnas, se expone el número de alumnos que acceden a cada centro en los intervalos de tiempo siguientes: alumnos a 5 minutos; a 10 minutos; a 20 minutos; a 30 minutos; a 40 minutos; y a 50 minutos (información esta última extraída mediante consultas a la base de datos citada anteriormente).

Con todos estos datos en la hoja de cálculo, el siguiente paso es identificar aquellos centros en donde el número de alumnos totales asignados es superior al de plazas ofertadas del resto (es decir, de centros «Sin límite» de tiempo de asignación). En el primer caso y para cada uno de ellos, se identifica el intervalo de tiempo en el que la suma de alumnos asignados más se acerca (sin superarla) al número de plazas ofertadas (celdas en negrita). Seguidamente se calcula el incremento de alumnos por minuto (dato que aparece en la columna «Dif./ min.interv» de la tabla 2). Este dato es clave para determinar el tiempo límite de la zona de asignación para cada centro con límite de plazas. El proceso de cálculo de este límite de zona temporal es el siguiente:

$$
\operatorname{Dif}_{\text {min. }_{\text {. }}}=\frac{\text { alums }_{T . \text { sup. }}-\text { alums }_{T . \text { lim. }}}{T \cdot_{\text {sup. }}-T_{\text {lim. }}}
$$

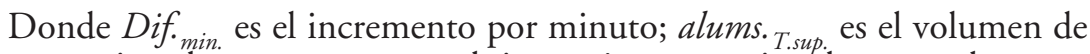
alumnos asignados a un centro en el tiempo justo superior al remarcado como

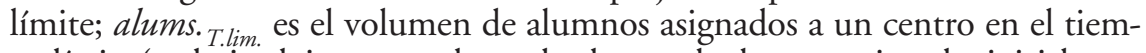
po límite (es decir, el tiempo en el que el volumen de alumnos asignados inicialmente es el que más se acerca, sin pasarse, al número de plazas ofertadas); y $T_{\text {sup. }}-T_{\cdot \text { lim. }}$. es la diferencia de tiempos en los que se consideran los volúmenes del numerador de la expresión. A continuación se calcula qué valor de tiempo ha de incrementarse al tiempo límite inferior (celdas en negrita) para que el incremento de alumnos asignados sea igual al número de plazas ofertadas en cada centro. La expresión para obtener el valor de tiempo a incrementar es:

$$
\text { alums }_{\text {T.lim } .}+\left(\text { Dif }_{\cdot_{\text {min }}}{ }^{*} x_{c}\right)=\text { Plazas }_{c}
$$

Donde Dif. $_{\text {min }}$ es el incremento de alumnos asignados por minuto;

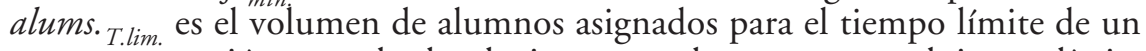
centro en cuestión; $x_{c}$ es el valor de tiempo que hay que sumar al tiempo límite para un centro $c$ (en el cual el número de alumnos previamente asignados más se acerca sin superarlo al número de plazas ofertadas); y Plazas es el número de plazas ofertadas para un centro $c$. Despejando $x_{c}$ de la ecuación se obtiene el 
Tabla 2. Asignación de escolares por centro de educación primaria, plazas y tiempo de acceso

\begin{tabular}{|c|c|c|c|c|c|c|c|c|c|c|c|c|}
\hline Nombre & Plazas & $\begin{array}{c}\text { Alum. } \\
\text { asignados }\end{array}$ & $5 \mathrm{~min}$ & $10 \min 2$ & $20 \min 3$ & $30 \min 4$ & $40 \min 5$ & $50 \mathrm{~min}$ & $\begin{array}{c}\text { Dif./ } \\
\text { min.interv }\end{array}$ & v Calculos & Limitecalc. & Limitemin. \\
\hline CEIP F. García Lorca & 250 & 267 & 93 & 267 & 267 & 267 & 267 & 267 & 35 & 250 & 10 & 9 \\
\hline CEIP A. Machado & 225 & 213 & 95 & 172 & 213 & 213 & 213 & 213 & & & Sin límite & Sin límite \\
\hline CEIP Trajano & 550 & 319 & 157 & 304 & 319 & 319 & 319 & 319 & & & Sin límite & Sin límite \\
\hline CEIP Suárez Somonte & 225 & 300 & 186 & 291 & 300 & 300 & 300 & 300 & 21 & 225 & 7 & 7 \\
\hline CEIP F. Giner de los Ríos & 450 & 236 & 112 & 216 & 236 & 236 & 236 & 236 & & & Sin límite & Sin límite \\
\hline CEIP Octavio Augusto & 475 & 483 & 164 & 296 & 483 & 483 & 483 & 483 & 19 & 475 & 20 & 19 \\
\hline CEIP Juan XXIII & 175 & 153 & 90 & 153 & 153 & 153 & 153 & 153 & & & Sin límite & Sin límite \\
\hline CEIP M. de Cervantes & 300 & 494 & 83 & 367 & 494 & 494 & 494 & 494 & 57 & 300 & 9 & 9 \\
\hline CEIP N. S. de la Antigua & 250 & 169 & 163 & 169 & 169 & 169 & 169 & 169 & & & Sin límite & Sin límite \\
\hline CEIP José María Calatrava & 225 & 298 & 152 & 213 & 283 & 283 & 283 & 283 & 7 & 225 & 12 & 12 \\
\hline CEIP Dion Casio & 300 & 326 & 131 & 326 & 326 & 326 & 326 & 326 & 39 & 300 & 9 & 9 \\
\hline CEIP F. Rguez. de la Fuente & 250 & 66 & 15 & 57 & 66 & 66 & 66 & 66 & & & Sin límite & Sin límite \\
\hline CEIP Pablo Neruda & 150 & 78 & 48 & 68 & 78 & 78 & 78 & 78 & & & Sin límite & Sin límite \\
\hline CEIP Ciudad de Mérida & 225 & 319 & 28 & 168 & 280 & 287 & 302 & 302 & 11 & 225 & 15 & 15 \\
\hline Col. Santa Eulalia, Coop & 250 & 132 & 118 & 132 & 132 & 132 & 132 & 132 & & & Sin límite & Sin límite \\
\hline Col. Santa Eulalia & 450 & 198 & 196 & 198 & 198 & 198 & 198 & 198 & & & Sin límite & Sin límite \\
\hline Col. Josefinas & 450 & 947 & & 338 & 947 & 947 & 947 & 947 & 61 & 450 & 12 & 12 \\
\hline Col. San Juan Bosco & 225 & 215 & 159 & 207 & 207 & 207 & 209 & 215 & & & Sin límite & Sin límite \\
\hline Coop. docente Atenea & 450 & 101 & 40 & 101 & 101 & 101 & 101 & 101 & & & Sin límite & Sin límite \\
\hline Escuela Libre Paideia & 24 & 768 & & & 18 & 434 & 766 & 768 & 42 & 24 & 20 & 20 \\
\hline Col. San Luis & 225 & 235 & 12 & 235 & 235 & 235 & 235 & 235 & 45 & 225 & 10 & 10 \\
\hline \multirow[t]{2}{*}{ Col. Salesianos } & 525 & 236 & 84 & 140 & 236 & 236 & 236 & 236 & & & Sin límite & Sin límite \\
\hline & 6649 & 6554 & 2125 & 4419 & 5740 & 6162 & 6512 & 6521 & & & & \\
\hline
\end{tabular}

Fuente: elaboración propia.

valor de tiempo (minutos) que hay que incrementar al tiempo límite inferior (cuyo volumen de alumnos asignados están remarcados en la tabla 2), para obtener un volumen de asignación de alumnos a cada centro (considerando ya el incremento de alumnos por minuto) acorde con el número de plazas ofertadas en el mismo (columnas «Limitecalc.» y «Limitemin.» de la tabla 2).

El dato de tiempo límite es utilizado finalmente en el entorno SIG para generar áreas de influencia sobre la red urbana existente. Estas áreas contienen el volumen de alumnos calculado en los pasos anteriores teniendo en cuenta las limitaciones de plazas.

Para calcular el área de asignación inicial de cada centro, se considera como punto de partida su localización sobre la red urbana de Mérida (capa de tipo puntual) y el coste del desplazamiento en tiempo sobre la misma. Las zonas de asignación generadas toman el mismo nombre que los centros que representan. Los cálculos para estas zonas se realizan de forma desagregada por nivel educativo ofertado. Así, en el caso de centros de educación infantil y primaria, se consideran únicamente aquellos puntos donde existe población con edades que les hacen ser potencialmente asignados a un centro de dicho 
nivel educativo, junto a los centros que ofertan plazas para este mismo nivel (el resto de centros y puntos residenciales no entran en ese cálculo de zonas). Este mismo procedimiento se repite para centros de secundaria y bachillerato. Por consiguiente, cada punto del entramado urbano se asigna al centro educativo más próximo en tiempo asegurándose de que todos los puntos residenciales con población potencial para cada nivel educativo quedan dentro de una zona de asignación concreta.

En el caso de centros sin límite de plazas (es decir, cuyo cálculo de población a asignar por distancia al mismo sea muy inferior al de plazas ofertadas), se calcula la zona de asignación mediante la herramienta de superficie mínima (convex hull). De esta forma, todos los puntos cuya población está asignada a un centro sin límite de plazas quedan dentro de su área de asignación, lo que minimiza la superficie de ocupación de dicha área.

Existe un tercer caso de alumnos que quedan fuera de los dos tipos de áreas de asignación anteriores. Es el caso de alumnos cuyas residencias quedan más alejadas de las zonas de asignación iniciales (porque los límites de tiempo han generado áreas que les dejan fuera) y también de las secundarias (zonas de asignación generadas para albergar alumnos cercanos a centros cuya demanda no exceda el número de plazas ofertadas). En estos casos, se lleva a cabo la reasignación de estos puntos residenciales y alumnos entre aquellos centros cuya oferta de plazas aún no esté completamente cubierta, considerando los siguientes criterios de asignación: minimizar el desplazamiento en vehículo privado y evitar rutas de viaje a través de zonas de la ciudad que resulten problemáticas para la movilidad general en hora punta.

Tras aplicar la metodología de asignación propuesta, todos los alumnos quedan inscritos en un centro educativo cuyo coste de desplazamiento es mínimo, y en un $90 \%$ el modo de desplazamiento más eficiente y efectivo es el peatonal.

\section{Análisis y resultados}

En este capítulo se muestran los principales análisis y resultados obtenidos tras aplicar la metodología propuesta en este trabajo. Estos se dividen en dos subapartados: resultados referidos a la encuesta realizada a los alumnos y aquellos otros que tienen que ver con la propia propuesta de asignación.

\subsection{Hábitos de movilidad de los estudiantes}

Los resultados de las encuestas realizadas revelan valores similares en cuanto al modo de desplazarse de los alumnos hasta sus respectivos colegios e institutos. El coche y el desplazamiento peatonal presentan los valores más elevados, mientras que el resto de modos son poco representativos. El transporte colectivo, que aúna a su vez al autobús urbano y el interurbano, corresponde en su mayoría a los alumnos de secundaria procedentes de zonas rurales que tienen que cursar sus estudios en Mérida, lo que explica que sean estos alumnos (10 \% 
Figura 4. Modo de llegada por nivel educativo y tipo de gestión del centro

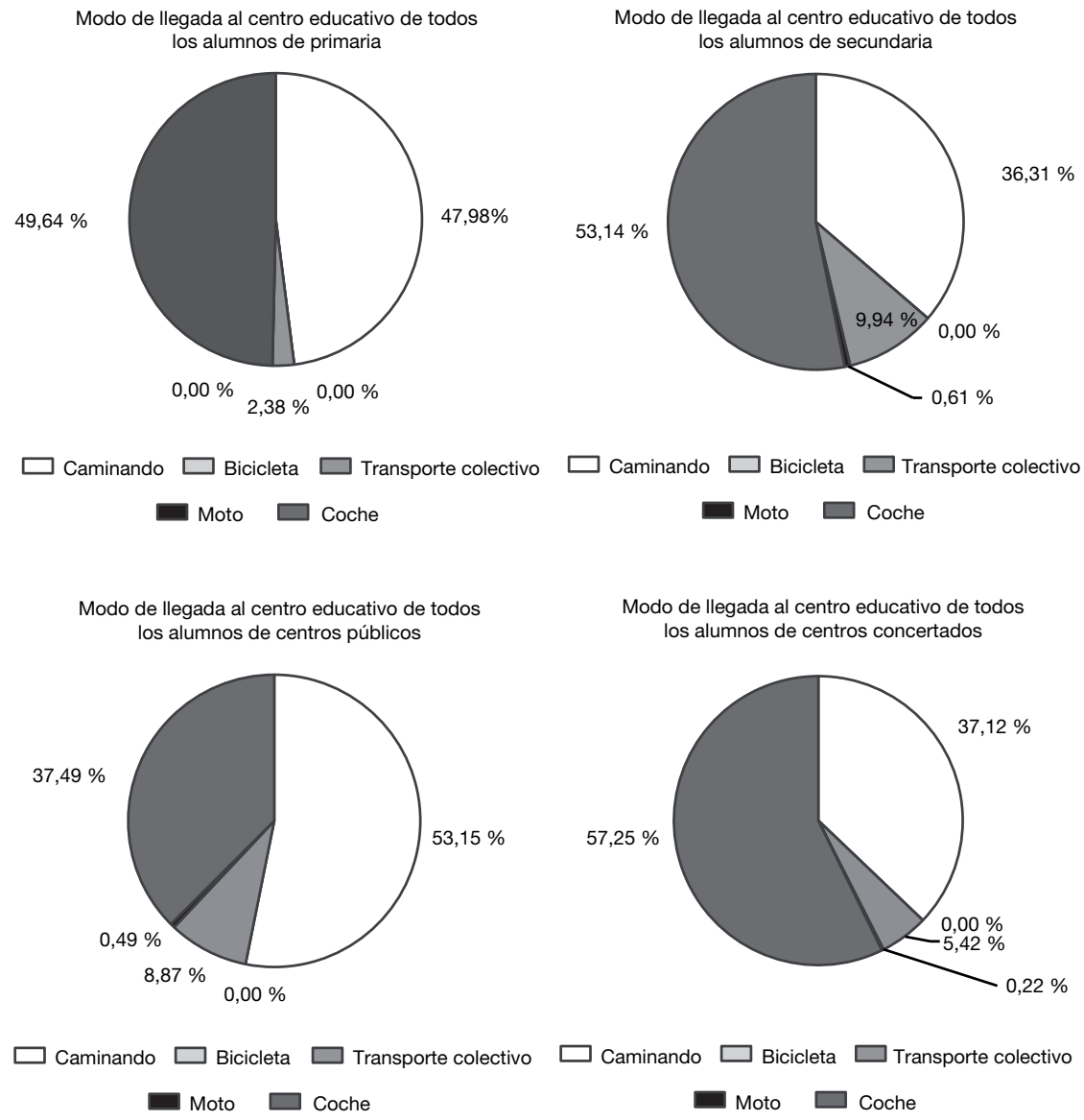

Fuente: elaboración propia.

aproximadamente) los usuarios fundamentales del transporte colectivo, claramente superior a los de educación primaria $(2,4 \%)$ (figura 4).

En cuanto al modo de transporte en función del tipo de gestión existente en cada centro, se observa una clara relación entre el desplazamiento en coche y el centro concertado, en detrimento del desplazamiento peatonal, más importante en los centros públicos.

Otro resultado interesante es la disparidad entre el modo realmente utilizado y el preferido, sobre todo en el caso de alumnos que cursan primaria (figura 5). Así, mientras que en los centros de secundaria los alumnos prefieren el modo de transporte que realmente utilizan, más de la mitad de los alumnos de la etapa de primaria prefieren desplazarse caminando en detrimento del vehículo privado (66 \%), lo que supone que aproximadamente un $18 \%$ de 
Figura 5. Modo de transporte preferido para llegar al centro educativo

Modo de desplazamiento que prefiere el total de los alumnos de primaria

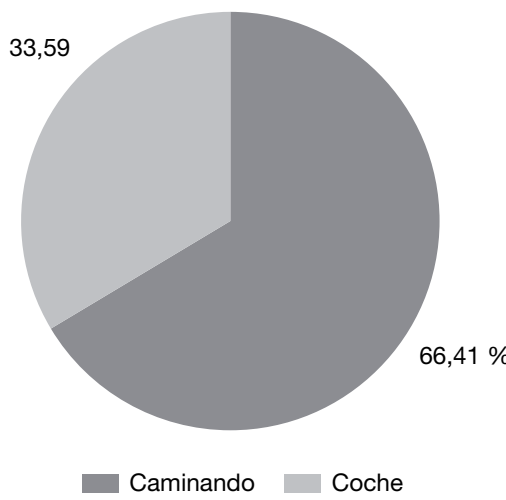

Modo de desplazamiento que prefiere el total de los alumnos de secundaria

$66,73 \%$

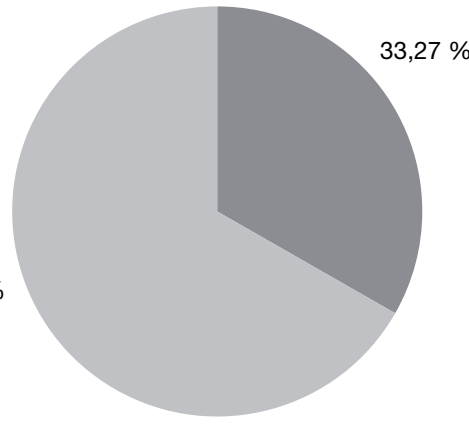

Caminando Coche

Fuente: elaboración propia.

estos se sienten obligados a moverse en automóvil en contra de su predisposición a hacerlo a pie.

Conocer la distancia entre la residencia del alumnado y su centro de estudio resulta fundamental para entender el tipo de movilidad que realiza. No obstante, ha resultado una variable difícil de conocer, fruto de la desorientación espacial de los jóvenes. Por ello, para medir esta distancia, sobre todo en el caso de alumnos de primaria, se limita la respuesta de la cuestión a: 1) cerca (si el estudiante vive en el mismo barrio donde está situado el centro) o 2) lejos (si el estudiante reside en un barrio diferente). Los resultados muestran claras diferencias en función del nivel educativo de los alumnos y de la gestión de los centros (figura 6). Así, mientras que en los públicos la mayoría del alumnado reside en el barrio donde se encuentra su centro, en el caso de los concertados lo hacen en un barrio diferente.

Diferentes estudios muestran que la distancia que un peatón en edad escolar está dispuesto a asumir para alcanzar un bien o un equipamiento es de menos de 1,6 kilómetros (DiGuiseppi et al., 1998; McDonald, 2007b) o de 10 minutos aproximadamente (Horning et al., 2008; Gutiérrez-Zornoza et al., 2014). Los resultados de la encuesta que muestran la relación entre el tiempo de llegada y el modo de desplazamiento (figura 7) revelan que, en los trayectos inferiores a los 10 minutos, en muchas ocasiones se sustituye la marcha a pie por el uso del coche sin una necesidad real más allá de la comodidad, tal como expresa el alumnado en repetidas ocasiones. Tal es así, que incluso un tercio de los desplazamientos de 0 a 5 minutos se realizan en coche.

Además, si se comparan los modos de transporte utilizados habitualmente por la población emeritense en el periodo de hora punta (coincidente con las entradas y salidas de los centros educativos) con los de la comunidad educa- 
Figura 6. Distancia desde el hogar al centro educativo en cuestión

Distancia del hogar al colegio (alumnos de primaria)

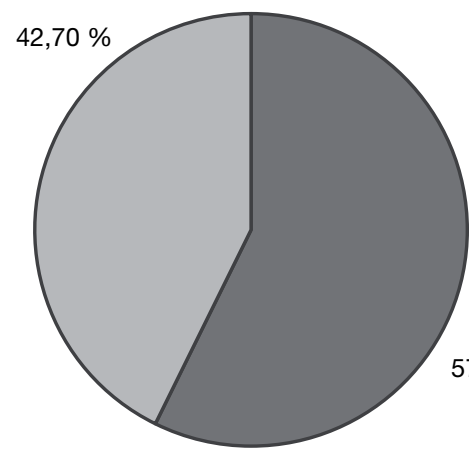

Cerca Lejos

Distancia del hogar al centro educativo (alumnos de centros públicos)

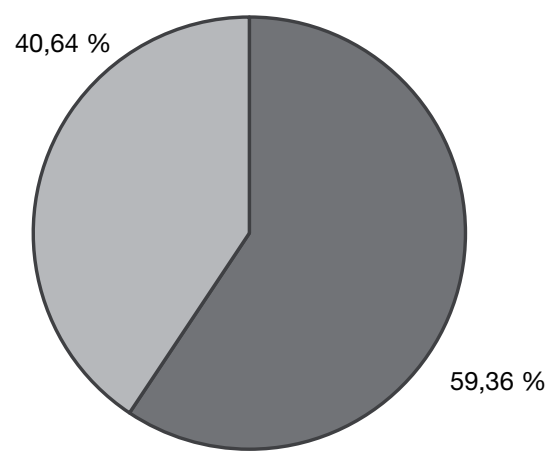

Cerca Lejos
Distancia del hogar al instituto (alumnos de secundaria)

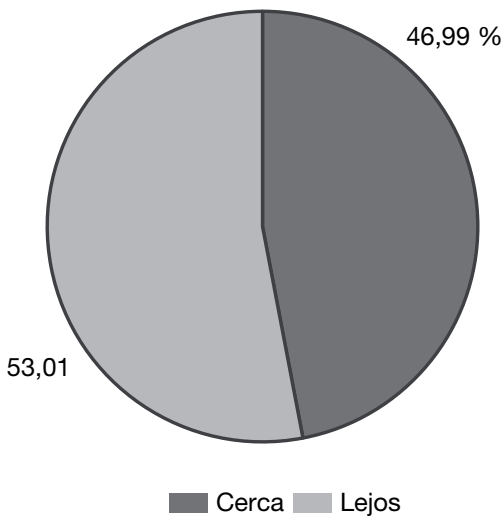

Distancia del hogar al centro educativo (alumnos de centros concertados)

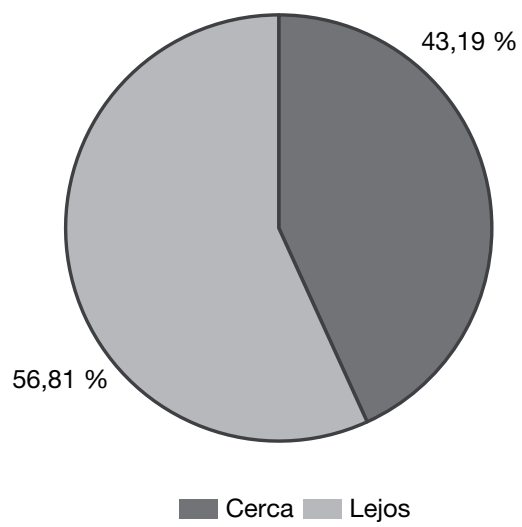

Fuente: elaboración propia.

tiva en particular (figura 8), se observan ciertas peculiaridades: mientras que más de la mitad de la población se decanta por el vehículo privado $(58 \%$ del total), muy por encima del desplazamiento peatonal (37\%), la comunidad escolar muestra una predilección similar hacia dos modos de desplazamiento: el desplazamiento peatonal ( $47 \%$ del total) y el vehículo privado (45\%). En ambos casos resulta residual el desplazamiento en moto, más si cabe en el caso de la comunidad educativa, por limitaciones de edad obvias (las licencias de ciclomotor no se consiguen hasta los quince años). También se observa un 
Figura 7. Modo de llegada al centro educativo en función de la distancia recorrida

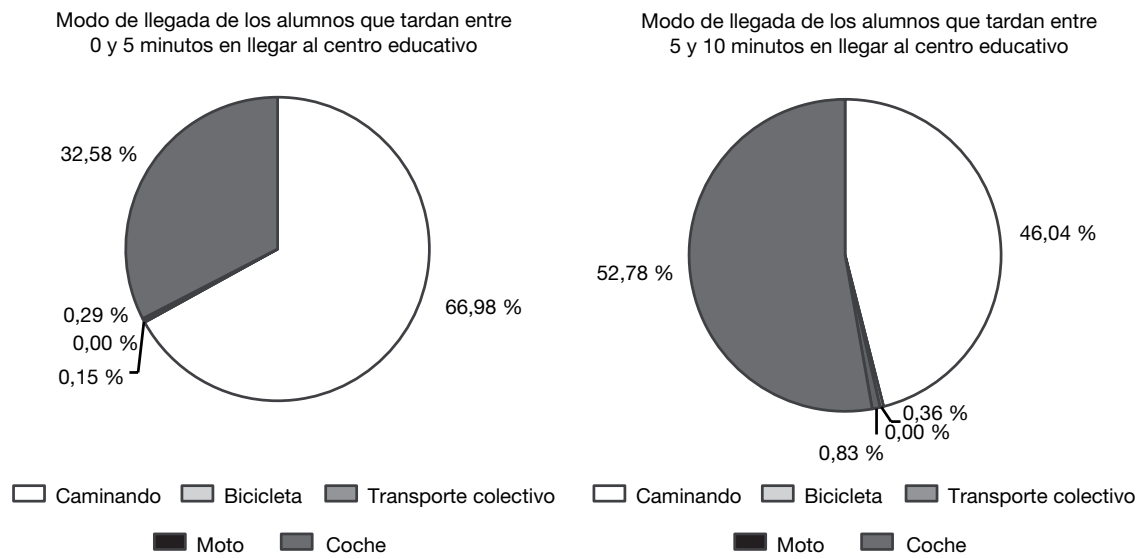

Fuente: elaboración propia.

Figura 8. Comparativa entre modos de los escolares y el resto de habitantes en HP

Modos de población emeritense (Ruiz, 2013)
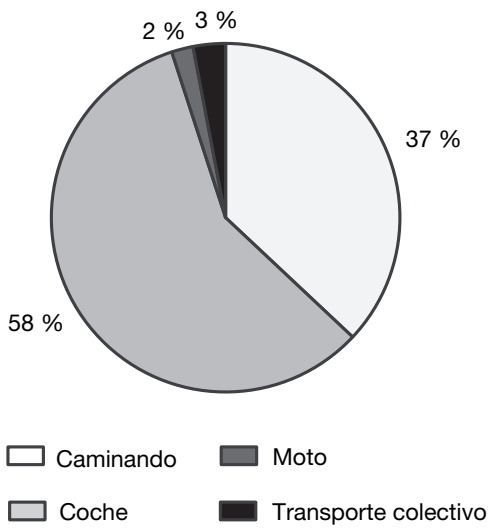

Modos de población escolar

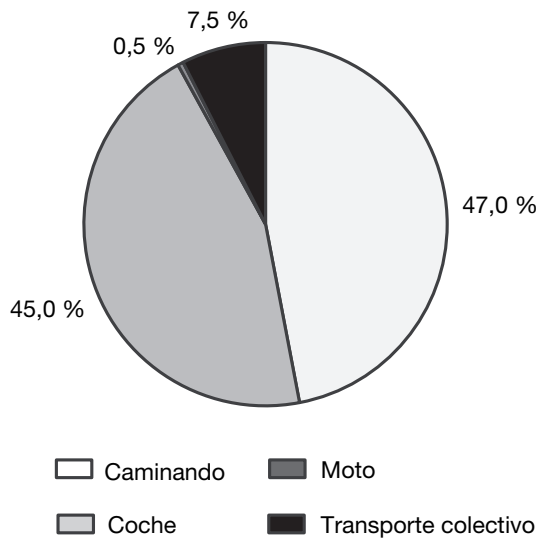

Fuente: elaboración propia.

incremento en el uso del autobús urbano en la comunidad escolar (el 7,5\% frente al $3 \%$ de la población emeritense). Ambos colectivos dan una enorme importancia al automóvil en sus accesos en hora punta (HP).

Si se tiene en cuenta la distribución de modos de transporte de los alumnos con respecto a la edad de los mismos (figura 9), se evidencian hábitos de movilidad heredados de sus progenitores. En cursos de $3^{\circ}$ a $5^{\circ}$ de educación primaria (alumnos con edades comprendidas entre los 8 y los 11 años), el modo de transporte predominante es el vehículo privado. Aunque son los alumnos 
Figura 9. Distribución por edad de modos de desplazamiento escolar

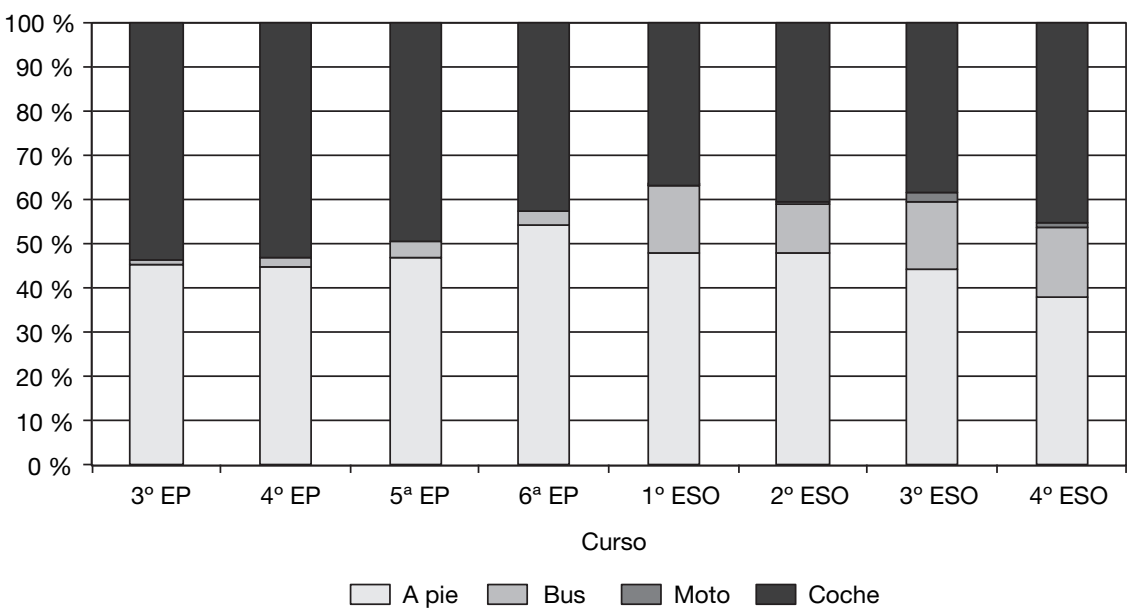

Fuente: elaboración propia.

con menor edad, tienen suficientemente desarrollada su competencia espacial como para hacer estos trayectos caminando (más si cabe cuando el vehículo privado no es el modo de transporte preferido por estos, según se muestra en la figura 5, y la distancia al mismo es considerada "cercana», figura 6). Este tipo de iniciativas ayudan al alumno a socializarse y a mejorar su percepción con el entorno y su orientación en el mismo a la vez que aumenta su autoestima.

Además, si se considera que en cursos posteriores (alumnos de ESO con edades comprendidas entre los 12 y los 16 años) los porcentajes de transporte público se reducen al autobús interurbano o servicio discrecional (utilizado por alumnos no residentes en Mérida), el vehículo privado sigue teniendo un lugar destacado entre el resto de modos, aún en desplazamientos de corta distancia (entre 5 y 10 minutos, figura 7).

Por tanto, si se pretende ofrecer una zonificación de asignación educativa que fomente un desplazamiento más sostenible en el acceso a los centros educativos, teniendo en cuenta que el uso del autobús urbano de Mérida es reducido (solo lo utiliza el $10 \%$ de la población emeritense y sus horarios de paso en paradas cercanas no presentan horarios compatibles), que buena parte de la población escolar reside a menos de diez minutos de un centro educativo, y que las dimensiones de la ciudad favorecen el uso de modos no motorizados, resulta clave el desplazamiento a pie en la propuesta metodológica que se presenta.

\subsection{Propuestas de asignación}

A la luz de los resultados obtenidos tras la encuesta, se propone una zonificación de asignación del alumnado a los diferentes centros educativos. Esta contribuye a racionalizar la movilidad urbana, garantizar la seguridad vial, 
fomentar la educación para la salud y la convivencia y reducir la distancia de los desplazamientos en el acceso diario a estos entornos. Así, se incrementa el espacio público y la autonomía del peatón, en general, y de la población joven que accede a los centros educativos, en particular.

Para ello se propone un nuevo diseño de las áreas de asignación de alumnado a los diferentes centros y niveles educativos ofertados en la ciudad, primando el factor de proximidad sobre el resto de factores de delimitación y considerando la ubicación real de los alumnos que potencialmente pueden acceder a dichos centros educativos (figuras 10 y 11). Frente a la zonificación actual que divide a la ciudad en cuatro macrozonas para los niveles de infantil y primaria y tan solo en dos (margen izquierdo y derecho del río Guadiana) para el nivel de secundaria, la propuesta que se presenta incrementa el número de zonas pero reduce considerablemente el tiempo de desplazamiento al centro asignado para la inmensa mayoría de los alumnos.

Vinculada con la zonificación propuesta, una intervención clave es el diseño de itinerarios escolares seguros en el acceso a los centros educativos. Teniendo en cuenta que en Mérida el $90 \%$ de la población escolar está a menos de diez minutos caminando del colegio (figura 12), la instauración de estos itinerarios ofrece una ruta segura para la que previamente se ha de hacer un reconocimiento del itinerario, contar con la colaboración de los negocios del entorno (muy

Figura 10. Propuesta de zonas de asignación a los centros de educación infantil y primaria

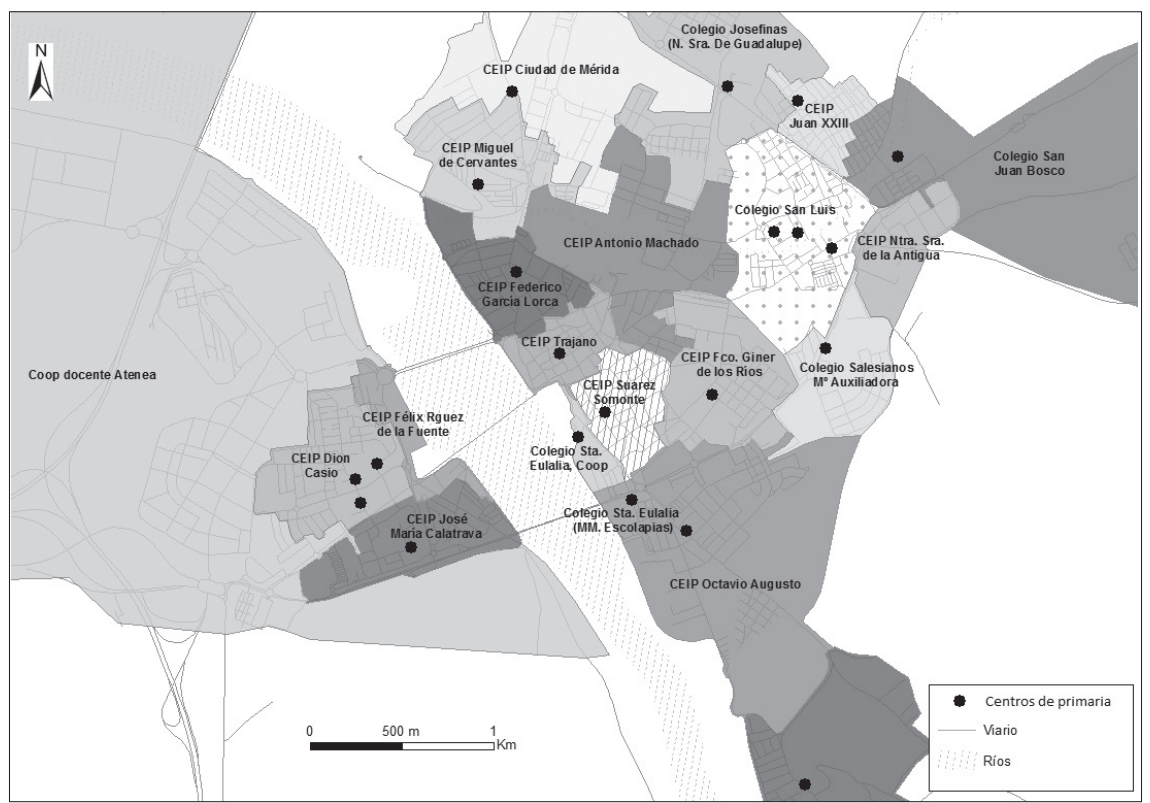

Fuente: elaboración propia. 
Figura 11. Propuesta de zonas de asignación a los centros de educación secundaria

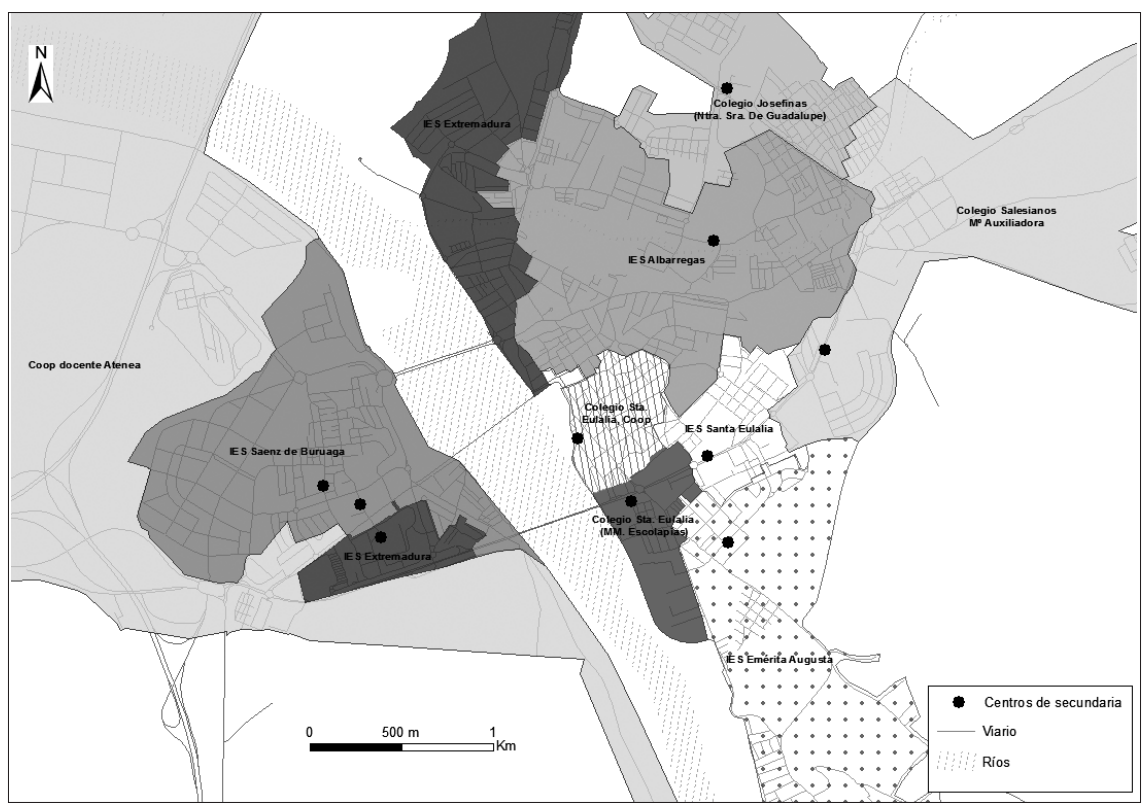

Fuente: elaboración propia.

Figura 12. Accesibilidad potencial a los centros educativos entre 0 y 10 minutos

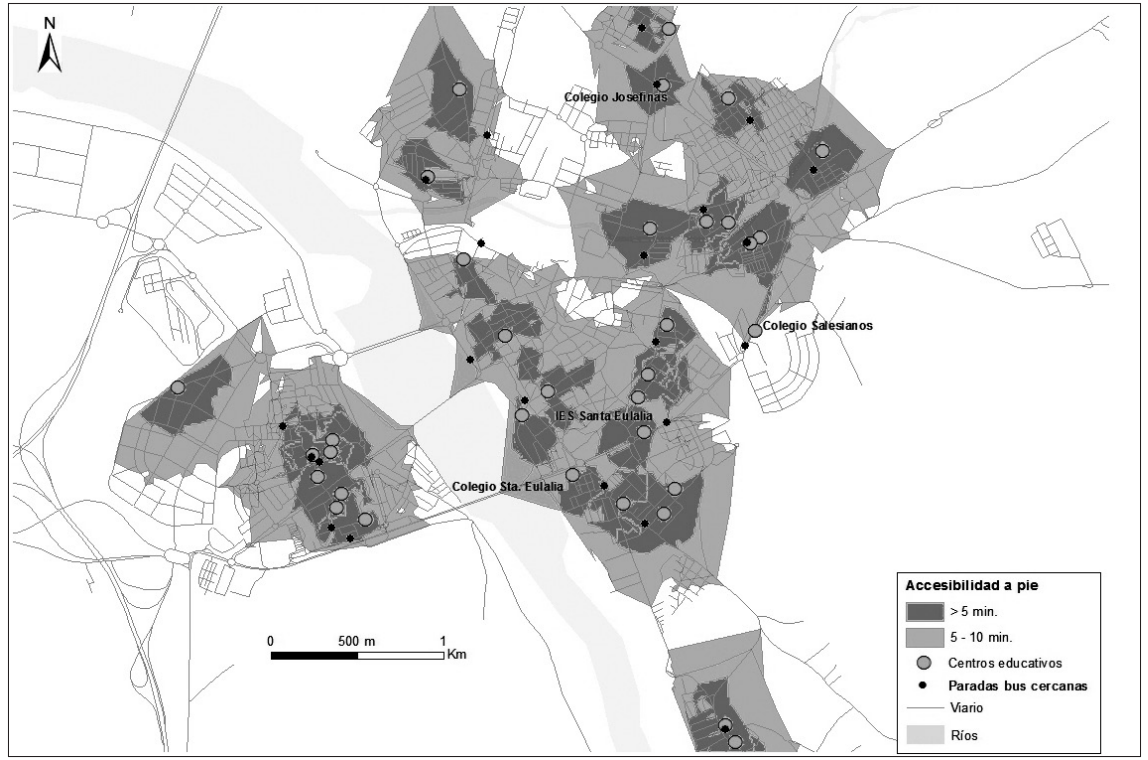

Fuente: elaboración propia.

92 Documents d'Anàlisi Geogràfica 2817, vol. 64/1 
útiles para guiar a chicos que pudieran desorientarse o para colocar carteles orientativos), disponer de voluntarios que acompañen a los alumnos en este desplazamiento e implicar a la familia.

\section{Discusión y conclusiones}

El análisis de las encuestas revela que los modos utilizados en mayor medida por los niños para desplazarse al centro educativo son el coche y el desplazamiento peatonal. El resto de modos resultan anecdóticos, incluyendo el transporte público. Este predominio del automóvil contrasta con otros estudios (Collins y Kearns, 2001; Boarnet et al., 2005; Wilson et al., 2010; Napier et al., 2011), donde se observa que la mayor parte de los alumnos acceden a centros educativos de primaria caminando. Esta diferencia puede deberse a unas distancias de recorrido más cortas propias de ciudades más densamente pobladas (grandes ciudades con más centros educativos mejor distribuidos).

Si se distingue entre niveles educativos y tipo de gestión, se observa que aquellos centros que disponen de un área de asignación de alumnos más pequeña (centros de educación primaria públicos) favorecen el desplazamiento en modos alternativos al automóvil (en este caso, el viaje a pie), mientras que los que disponen de un área más amplia fomentan el modo privado (educación secundaria o centros privados). Además, la percepción de libertad y estatus social de los alumnos de mayor edad con el uso del vehículo privado, unido a los hábitos de movilidad que han ido adquiriendo por parte de sus padres, conlleva un aumento de su uso en los centros de educación secundaria. Por contraste, el currículo de educación secundaria no recoge ningún objetivo educativo relacionado con la educación vial, a pesar de las más que evidentes conductas de inseguridad que muestran los adolescentes (Meneses et al. 2010).

Ello indica que el coche ha ido ganando terreno en la vida cotidiana del alumnado como modo de desplazamiento, que observa con normalidad su uso, independientemente del espacio recorrido y el tiempo utilizado en el desplazamiento. Esta idea se puede relacionar con la asunción del automóvil como garante del derecho a la movilidad en las sociedades avanzadas, aunque habría que preguntarse si sirve a todos los grupos sociales por igual. Algunas de las consecuencias del abusivo uso del vehículo privado son el incremento de la congestión, demoras, accidentes y pérdida de espacio público para el peatón, sin olvidar los problemas ambientales.

Por otra parte, el hecho de que el coche se haya convertido en un instrumento esencial en los desplazamientos urbanos genera un tipo de comportamiento a imitar en el futuro. Por ello es muy importante hacer entender a los jóvenes que hay otros modos de desplazamiento que ayudarían a acercarse a la idea de movilidad sostenible, a la vez que permiten desarrollar sus capacidades espaciales y de orientación.

Respecto a las preferencias de los alumnos a la hora de desplazarse, destaca el modo peatonal como el ideal elegido por la mayoría. Así, el porcentaje que desearían ir caminando al colegio es similar al de los que se desplazan a pie. 
No obstante, sí se observan diferencias en el uso del vehículo privado: es casi tres veces superior el número de alumnos que se desplazan en coche al colegio al de aquellos que realmente lo desearían.

En relación con los resultados relativos al tiempo de llegada a los centros educativos, el efecto de la motorización no ha contribuido ni a ganar tiempo ni calidad de vida, sino todo lo contrario. Uno de cada tres desplazamientos que realiza el alumnado de Mérida en coche se produce dentro del mismo barrio en un tiempo inferior a cinco minutos. Estos datos ponen de manifiesto el uso excesivo e innecesario del vehículo privado, que, además de generar atascos, priva a los alumnos de la adquisición de hábitos saludables que beneficiarían no solo al individuo sino también a la propia movilidad de la ciudad. Estos hábitos, unidos a costumbres como dejar a los estudiantes en la puerta de los centros o lo más cerca posible, generan congestión e inseguridad en las inmediaciones de colegios e institutos.

Por otro lado, respecto a la zonificación propuesta, esta presenta interesantes ventajas y potencialidades que deben tener muy en cuenta los decisores políticos: a) cada centro tendría asegurada su oferta y se evitaría la saturación de unos y la infrautilización de otros, $b$ ) se podría organizar la oferta educativa con suficiente antelación y no estaría supeditada a la posible demanda, $c$ ) existiría un menor grado de congestión y problemas de seguridad vial y d) se conseguiría una distribución más equitativa de los recursos de la ciudad. No obstante, como contrapartida, hay un elemento que debería considerarse y señalarse en la solución empleada cuando se supera la capacidad de los centros, y es que los estudiantes reasignados son los que quedan en las franjas más alejadas, es decir, que los que están ya en una situación poco adecuada empeoran al ser asignados a un centro que les queda aún más alejado. Pareciera que estos alumnos quedan abocados al empleo del vehículo particular, pero este hecho podría considerarse una oportunidad para potenciar o redelimitar rutas de autobús urbano que tengan por objetivo principal proporcionar servicios discrecionales eficientes a la comunidad educativa en un horario compatible.

Por último, con la puesta en marcha de los itinerarios escolares seguros propuestos y que facilitaría la nueva zonificación, se incrementaría la autonomía de los escolares al ofrecer nuevos espacios de aprendizaje en su entorno cotidiano y se crearían hábitos saludables de movilidad, con sus ventajas para la salud, el medio ambiente y una mejor socialización e integración en la comunidad que favorece el desarrollo de las habilidades cognitivas y de convivencia (Miras Martínez, 2000).

\section{Referencias bibliográficas}

Andersson, E., Malmberg, B. y Östh, J. (2012). «Travel-to-school distances in Sweden 2000-2006: changing school geography with equality implications». Journal of Transport Geography, 23, 35-43. $<$ https://doi.org/10.1016/j.jtrangeo.2012.03.022>

ANDRE-BeCHElY, L. (2007). "Finding space and managing distance: public school choice in an urban California district». Urban Studies, 44, 1355-1376. <https://doi.org/10.1080/00420980701302304> 
BARR, S. y PRILLWITZ, J. (2012). «Green travellers? Exploring the spatial context of sustainable mobility styles». Applied Geography, 32, 798-809. <https://doi.org/10.1016/j.apgeog.2011.08.002>

Boarnet, M. G., Anderson, C. L., Day, K., Mcmillan, T. y Alfonzo, M. (2005). «Evaluation of the California Safe Routes to School legislation, urban form changes y children's active transportation to school». American Journal of Preventive Medicine, 28, 134-140.

<https://doi.org/10.1016/j.amepre.2004.10.026>

BocareJo, J. P. y Oviedo, D. R. (2012). "Transport accessibility y social inequities: a tool for identification of mobility needs and evaluation of transport investments». Journal of Transport Geography, 24, 142-154. <https://doi.org/10.1016/j.jtrangeo.2011.12.004>

Broberg, A. KytTÄ, M. y FAGERhOLM, N. (2013). «Child-friendly urban structures: Bullerby revisited». Journal of Environmental Psychology, 35, 110-120. <https://doi.org/10.1016/j.jenvp.2013.06.001>

Brown, B., Mackett, R., Gong, Y., Kitazawa, K. y Paskins, J. (2008). «Gender differences in children's pathways to independent mobility». Children's Geographies, 6, 385-401. <https://doi.org/10.1080/14733280802338080>

BrueCKNeR, J. K. (2000). «Urban Sprawl: diagnosis and remedies». International Regional Science Review, 23, 160-171. <https://doi.org/10.1177/016001700761012710>

Bunar, N. y Kallstenius, J. (2005). In My Old School I Learnt The Wrong Swedish Language. A Study of Free School Choice in the Polarised Urban Space. Estocolmo: Integrationsverket.

Butler, T., Hamnett, C., Ramsden, M. y Webber, R. (2007). «The best, the worst and the average: secondary school choice and education performance in East London». Journal of Education Policy, 22, 7-29. <https://doi.org/10.1080/02680930601065718>

Cameron, I., KenWorthy, J. R. y Lyons, T. J. (2003). «Understanding and predicting private motorized urban mobility». Transportation Research Part D, 8, 267-283. <https://doi.org/10.1016/S1361-9209(03)00003-8>

Cardozo, O. D., García-Palomares, J. C. y Gutiérrez, J. (2012). «Application of geographically weighted regression to the direct forecasting of transit ridership at station-level». Applied Geography, 34, 548-558. <https://doi.org/10.1016/j.apgeog.2012.01.005>

CHEN, J., SHAW, S. L., YU, H., LU, F., CHAI, Y. y JiA, Q. (2011). «Exploratory data analysis of activity diary data: a space-time GIS approach». Journal of Transport Geography, 19, 394-404. <https://doi.org/10.1016/j.jtrangeo.2010.11.002>

Collins, D. C. A. y Kearns, R. A. (2001). "The safe journeys of an enterprising school: negotiating landscapes of opportunity y risk». Health \& Place, 7, 293-306. <https://doi.org/10.1016/S1353-8292(01)00021-1>

Commission of THE European Communities (2006). Communication from the Commission to the Council and the European Parliament - Keep Europe moving - Sustainable mobility for our continent - Mid-term review of the European Commission's 2001 Transport White Paper. \{SEC (2006)768\} COM/2006/0314 final. Bruselas. 
- (2007). Green Paper: Towards a new culture for urban mobility [COM(2007)55]. Bruselas.

- (2011). White Paper Roadmap to a Single European Transport Area - Towards a competitive and resource efficient transport system. COM/2011/0144 final. Bruselas.

DAVIS, A, y Jones, L. (1997). «Whose neighbourhood? Whose quality of life? Developing a new agenda for childrens health in urban settings». Health Education Journal, 56, 350-362. <https://doi.org/10.1177/001789699705600404>

DEKA, D. (2013). «An explanation of the relationship between adults' work trip mode and children's school trip mode through the Heckman approach». Journal of Transport Geography, 31, 54-63. <https://doi.org/10.1016/j.jtrangeo.2013.05.005>

DiGuiseppi, C., RoberTs, I., Li, L. y Allen, D. (1998). «Determinants of car travel on daily journeys to school: cross sectional survey of primary school children». British Medical Journal, 316, 1426-2428.

Dombriz, M. A. (2009). "Urbanismo y movilidad: dos caras de la misma moneda». Ingeniería y Territorio, 86, p. 4-9.

Fotel, T. y Thomsen, T. U. (2004). «The surveillance of children's mobility». Surveillance \& Society, 14, 535-554.

Fox, K. R. (2004). "Childhood obesity and the role of physical activity». Journal of the Royal Society for the Promotion of Health, 124, 34-39. <https://doi.org/10.1177/146642400312400111>

FYHRI, A. (2002). Barns reiser til skolen. En spørreundersøkelse om reisevaner og trafikksikkerhet pa skoleveien. Report 616. Oslo: Transportøkonomisk Institutt.

Fyhri, A., Hjorthol, R., Mackett, R. L., Fotel, T. N. y KytTÄ, M. (2011). "Children's active travel and independent mobility in four countries: development, social and contributing trends and measures». Transport Policy, 18, 703-710. <https://doi.org/10.1016/j.tranpol.2011.01.005>

Gutiérrez, J. A. y Pedrazo, F. (2010). Proyecto piloto municipal para la promoción de la movilidad sostenible de Mérida. Estudio de tráfico viario. Ref. 268/08. Mérida: Ministerio de Fomento. Inédito.

Gutiérrez-Zornoza, M., Rodríguez-Martín, B., MartíneZ-Andrés, M., GarcíaLÓPEZ, U. y SÁNCHEZ-LÓPEZ, M. (2014). «Percepción del entorno para la práctica de actividad física en escolares de la provincia de Cuenca, España». Gaceta Sanitaria, 28, 34-40.

<https://doi.org/10.1016/j.gaceta.2013.04.011>

Hadayeghi, A., Shalaby, A. S. y Persaud, B. N. (2003). «Macrolevel accident prediction models for evaluating safety of urban transportation systems». Transportation Research Board, 1840, 87-95. $<$ https://doi.org/10.3141/1840-10>

Horning, J., El-GENEIDY, A. M., y KRIZEK, K. J. (2008). «Perceptions of walking distance to neighborhood retail and other public services». En: 87th Meeting of the Transportation Research Board. Washington DC.

Jensen, A. M., Ben-Arieh, A., Conti, C., Kutsar, D., Phadraig, M. N. G. y Nielsen, H. W. (ed.) (2004). Trondheim: Norwegian Centre for Child Research.

Jensen, S. U. (2008). "How to obtain a healthy journey to school». Transportation Research Part A, 42, 475-486. <https://doi.org/10.1016/j.tra.2007.12.001> 
Karsten, S., Ledoux, G., Roeleveld, J., Felix, C. y Elshof, D. (2003). «School choice and ethnic segregation». Educational Policy, 17, 452-477. <https://doi.org/10.1177/0895904803254963>

Kerr, J., Rosenberg, D., Sallis, J. F., Saelens, B. E., Frank, L. D. y Conway, T. L. (2006). "Active commuting to school: associations with environment and parental concerns». Medicine and Science in Sports and Exercise, 38, 787-793. <https://doi.org/10.1249/01.mss.0000210208.63565.73>

КүтTё, M. (2008). Children in outdoor contexts: affordances and independent mobility in the assessment of environmental child friendliness. Saarbrücken: VDM Verlag Dr. Müller.

MACKETT, R. L. (2002). «Increasing car dependency of children. Should we be worried?». Municipal Engineer, 151, 29-38.

- (2003). The effectiveness of initiatives to reduce children's car use. Estrasburgo: Association for European Transport.

Mackett, R. L., Lucas, L., Paskins, J. y Turbin, J. (2005). «The therapeutic value of children's every day travel». Transportation Research Part A, 39, 205-219. <https://doi.org/10.1016/j.tra.2004.09.003>

Martin, S. y CARLSON, S. (2005). «Barriers to children walking to or from school: United States, 2004». Morbidity and Mortality Weekly Report, 54, 949-952.

MCDONALD, N. C. (2007a). «Active transportation to school - trends among US schoolchildren, 1969-2001». American Journal of Preventive Medicine, 32, 509-516. <https://doi.org/10.1016/j.amepre.2007.02.022>.

- (2007b). "Children's mode choice for the school trip: the role of distance and school location in walking to school». Transportation, 35, 23-35. <https://doi.org/10.1007/s11116-007-9135-7>

MCMillan, T. E. (2007). "The relative influence of urban form on a child's travel mode to school». Transportation Research, Part A: Policy y Practice, 41, 69-79. <https://doi.org/10.1016/j.tra.2006.05.011>

Meneses Falcón, C., Gil García, E., y Romo Avilés, N. (2010). «Adolescentes, situaciones de riesgo y seguridad vial». Atención Primaria. Publicación Oficial de la Sociedad Española de Medicina de Familia y Comunitaria, 42, 452-458. <https://doi.org/10.1016/j.aprim.2009.10.013>

MinisTERIO DE FOMENTO (2007): Encuesta de movilidad de las personas residentes en España (MOVILIA 2006/2007). Madrid.

Miras Martínez, F. (2000). «Los trastornos del desarrollo intelectual». En: YUSTE Rossell, N. (ed.). Educación vial en el ámbito juvenil. Almería: Universidad de Almería, 145-155.

Monzón, A. (2005): «Gestión del transporte metropolitano». Gobernar las Metrópolis, 519, 405-434.

Napier, M. A., Brown, B. B., Werner, C. M. y Gallimore, J. (2011). «Walking to school: Community design and child and parent barriers». Journal of Environmental Psychology, 31, 45-51. <https://doi.org/10.1016/j.jenvp.2010.04.005>

Ortúzar, J. D. y Willumsen, L. (2008). Modelos de transporte. Santander: PubliCan. Universidad de Cantabria.

Pozueta, J. y Gurovich, A. (2007). Alternativas al modelo dominante de ciudad dispersa, zonificada y de baja densidad: el caso de los corredores fluviales y la interfaz urbana rural de Madrid y Santiago de Chile. Madrid: AECI, Ministerio de Asuntos Exteriores y de Cooperación de España. Proyecto A/4930/06. 
Roberts, I., Norton, R. T. y TAuA, B. (1996). «Child pedestrian injury rates: the importance of exposure to risk relating to socioeconomic and ethnic differences, in Auckland, New Zealand». Journal of Epidemiology and Community Health, 50, 162-165. <https://doi.org/10.1136/jech.50.2.162>.

Roberts, I., Carlin, J., Bennett, C., Bergstrom, E., Guyer, B., Nolan, T., NorTOn, R., Pless, I. B. y Stevenson, M. (1997). "An international study of the exposure of children to traffic». Injury Prevention, 3, 89-93. $<$ https://doi.org/10.1136/ip.3.2.89>

RuIZ, E. (2013). Metodología para la determinación de la movilidad en ciudades de tamaño medio. El caso de Mérida. Cáceres: Universidad de Extremadura. Tesis doctoral.

Salmon, J., Timperio, A., Cleland, V. y Venn, A. (2005). "Trends in children's physical activity and weight status in high and low socio-economic status areas in Melbourne, Victoria, 1985-2001». Australian and New Zealand Journal of Public Health, 29, 337-342. <https://doi.org/10.1111/j.1467-842X.2005.tb00204.x>.

SANG, S., O'Kelly, M. y KWAN, M. P. (2011). «Examining commuting patterns». Urban Studies, 48, 891-909. <https://doi.org/10.1177/0042098010368576>

Seguí, J. M. y MARTíneZ, M. R. (2004). «Los sistemas inteligentes de transporte y sus efectos en la movilidad urbana e interurbana». Scripta Nova, 6, 170.

STEG, L. y GIFFORD, R. (2005). «Sustainable transportation and quality of life». Journal of Transport Geography, 13, 59-59. <https://doi.org/10.1016/j.jtrangeo.2004.11.003>

Trumberg, A. (2011). Divided schools. Processes of Segregation in the Swedish School System. Örebro: Örebro Universitet.

WALFORD, G. (2003). «School choice and educational change in England and Wales». En: Plank D. N. y SyKes, G. (eds.). Choosing Choice. School Choice in International Perspective. Amsterdam y Nueva York: Teachers College Press, 68-92.

Wilson, E. J., Marshall, J., Wilson, R. y KrizeK, K. J. (2010). «By foot, bus or car: children's school travel and school choice policy». Environment and Planning $A, 42,2168-2185$.

<https://doi.org/10.1068/a435> 